\title{
Application of optical phase lock loops in coherent beam combining
}

\section{Wei Liang, Naresh Satyan, Firooz Aflatouni, Amnon Yariv, Anthony Kewitsch, et al.}

Wei Liang, Naresh Satyan, Firooz Aflatouni, Amnon Yariv, Anthony Kewitsch, George Rakuljic, Hossein Hashemi, "Application of optical phase lock loops in coherent beam combining," Proc. SPIE 6873, Fiber Lasers V: Technology, Systems, and Applications, 68731Y (25 February 2008); doi: 10.1117/12.764378

SPIE Event: Lasers and Applications in Science and Engineering, 2008, San Jose, California, United States 


\title{
Application of Optical Phase Lock Loops in Coherent Beam
}

\section{Combining}

\author{
Wei Liang, ${ }^{\mathrm{a}}$ Naresh Satyan, ${ }^{\mathrm{a}}$ Firooz Aflatouni, ${ }^{\mathrm{c}}$ Amnon Yariv, ${ }^{\mathrm{a}}$ Anthony Kewitsch, ${ }^{\mathrm{b}}$ George \\ Rakuljic, ${ }^{b}$ and Hossein Hashemic ${ }^{\mathrm{c}}$ \\ ${ }^{a}$ Department of Applied Physics and Department of Electrical Engineering, MC 128-95, California \\ Institute of Technology, Pasadena, CA 91125; \\ ${ }^{\mathrm{b}}$ Telaris Inc., 2118 Wilshire Blvd. \#238, Santa Monica, CA 90403; \\ ${ }^{c}$ Department of Electrical Engineering - Electrophysics, University of Southern California, Los \\ Angeles, CA 90089
}

\begin{abstract}
Coherent Beam Combining (CBC) technology holds the promise of enabling laser systems with very high power and near-ideal beam quality. In this work we propose and demonstrate a novel CBC servo system using optical phase lock loops for phase control. This servo system is based on entirely electronic components and, consequently, can be considerably more compact and less expensive compared to servo systems made of optical phase/frequency shifters. In the proof-of-concept experiments we have combined two $100 \mathrm{~mW} 1064 \mathrm{~nm}$ commercial semiconductor lasers with the filled-aperture approach at an efficiency of $94 \%$ and also two $50 \mathrm{~mW} 1538 \mathrm{~nm}$ commercial semiconductor lasers using the tiled-aperture approach with a strehl ratio of 0.9. In addition, we also present a theoretical consideration of the influence of various sources of noise on the combining efficiency of a cascaded filled-aperture CBC system.
\end{abstract}

Keywords: Coherent beam combining, optical phase lock loops

\section{INTRODUCTION}

Lasers with high average power and high beam quality have a large potential in industrial applications and scientific research, and have been sought after since the invention of the laser. Recently, fiber lasers/amplifiers have been attracting a good deal of attention due to their high output power, efficiency and near diffraction-limited beam quality [See www.ipgphotonics.com]. Further increase of the power available from a single-mode fiber will be ultimately limited by nonlinear effects and material damage. Coherent beam combining provides a promising approach to obviate this problem and thus to further scale up the power of a single optical beam by tens or hundreds of times without degrading the spectral purity and the beam quality[1-4]. By definition, coherent beam combining (CBC) requires all the beams to have the same frequency and a stable relative phase. To achieve high combining efficiency and high beam quality, precise control of the relative phase, amplitude, polarization, and pointing of the beams are required[2]. Among all the factors affecting the combining efficiency, the relative phase remains the most critical and difficult to control. So far $\mathrm{CBC}$ has been implemented using common resonator[5], evanescent-wave coupling, self-organizing[4], injection locking[1] and active feedback[6,7] mechanisms. In the active feedback approach, the variation of the differential optical path lengths of the combining beams is detected and fed back to a servo system which maintains a constant relative phase between the beams. In previously demonstrated work, the phase actuator of the servo system is either an optical phase modulator[1, 6], a piezo fiber stretcher[8],or an acoustic optical modulator (AOM)[7].

In this paper we propose and demonstrate a novel approach to control the frequency and phase of the individual beams using multiple optical phase lock loops(OPLLs) [9-11]. In this approach, an array of semiconductor lasers is phaselocked to a common master laser using current injection heterodyne OPLLs. Once they are made mutually coherent they can be amplified using high power fiber gain units, and then coherently combined using either a filled-aperture or a tiledaperture implementation. In a heterodyne OPLL, the phase of the slave laser can be adjusted by the RF offset signal. Hence, instead of using an optical phase shifter, one can use an electric phase shifter or frequency shifter (such as a Voltage Controlled Oscillator) to compensate for variations in the optical path lengths of the combining beams.

Fiber Lasers V: Technology, Systems, and Applications, edited by Jes Broeng, Clifford Headley, Proc. of SPIE Vol. 6873, 68731Y, (2008) -0277-786X/08/\$18 - doi: 10.1117/12.764378 
Compared to previously demonstrated approaches using optical phase or frequency shifters, this approach enables a much cheaper, more compact, fully electronic servo system.

\section{OPTICAL PHASE LOCK LOOPS: THEORY AND EXPERIMENT}

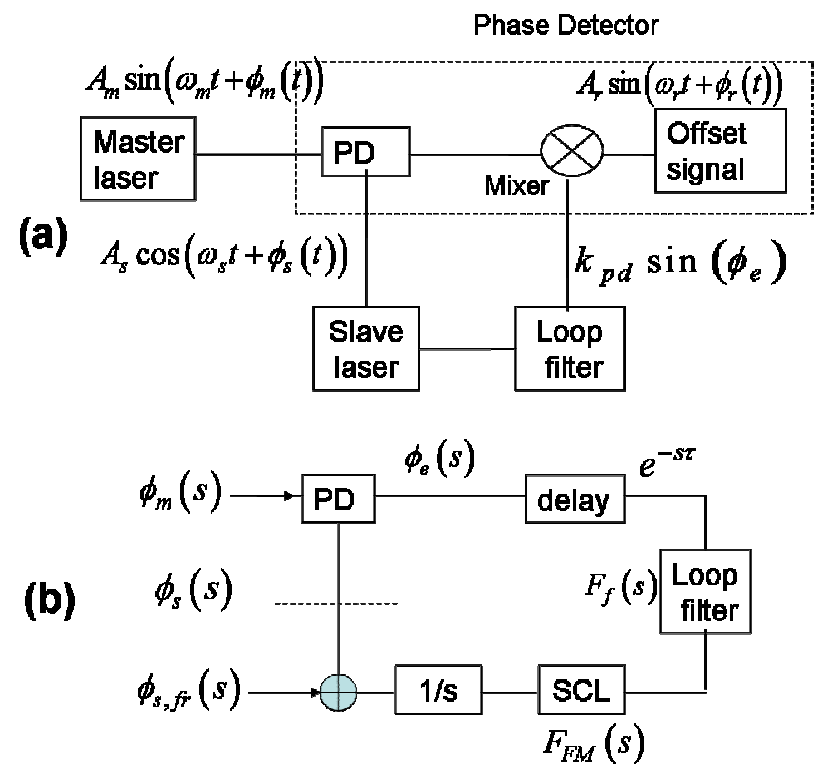

Fig. 1(a) Schematic diagram of a heterodyne OPLL. (b) Small signal linearized model of the OPLL.

Due to its well known current frequency-modulation(FM) property, a semiconductor laser(SCL) can act as a current controlled oscillator, and thus be phase-locked to a master laser using an OPLL[12-14]. A schematic diagram of a heterodyne OPLL is given in Fig. 1(a). Offset by a RF signal, the frequency and phase difference between the master laser and the slave laser is detected by the phase detector. The phase error signal $k_{p d} \sin \phi_{e}$ is shaped by a loop filter and fed back into the slave laser to complete the feedback loop. When the loop is in lock, the slave laser's frequency/phase $\omega_{s}, \phi_{s}$ and the steady-state phase error $\phi_{e 0}$ satisfy

$$
\begin{aligned}
& \omega_{s}=\omega_{m}-\omega_{r}, \quad \phi_{s}=\phi_{m}-\phi_{r}-\phi_{e 0} \\
& \sin \left(\phi_{e 0}\right)=\left(\omega_{m}-\omega_{s, f r}-\omega_{r}\right) / K_{d c}
\end{aligned}
$$

where $\omega_{m}, \omega_{s, f r}, \omega_{r}$ are respectively the frequency of the master laser, the free-running slave laser and the RF offset signal, $\phi_{m}, \phi_{s}, \phi_{r}$ are, respectively, the phase of the master laser, the locked slave laser and the RF offset signal. Eq. (1) indicates that the slave laser tracks the frequency and phase of the master laser with a frequency and phase offset determined by the RF offset signal. If a number of SCLs are phase-locked to the same master laser and are offset by the same RF offset signal, all of them will possess the same frequency and phase and thus can be coherently combined. The loop performance and phase noise of an OPLL can be studied in the frequency domain with a linearized model based on the small signal perturbation approximation[15], The phase noise of the master laser and the slave laser are contained in the variables $\phi_{m}$ and $\phi_{s, f r}$. The phase noise of the RF source and the shot noise of the photodetector (PD) are neglected compared to the SCLs' phase noise.

Following the standard PLL analysis[15], the differential phase error between the slave and the master lasers, and the phase of the locked slave laser are

$$
\begin{gathered}
\phi_{e}(s)=\left(\phi_{m}(s)+\phi_{s, f r}(s)\right) \cdot H_{e}(s) \\
\phi_{s}(s)=\phi_{m}(s) \cdot H_{o}(s)+\phi_{s, f r}(s) \cdot H_{e}(s) \\
\text { where } \quad H_{o}(s)=\frac{\partial \phi_{s}}{\partial \phi_{m}}=\frac{G_{o p}(s)}{1+G_{o p}(s)}, \quad H_{e}(s)=\frac{\partial \phi_{e}}{\partial \phi_{m}}=\frac{1}{1+G_{o p}(s)}
\end{gathered}
$$


are respectively the signal transfer function and the error transfer function[15], and

$$
G_{o p}(s)=\frac{K_{d c} \cos \phi_{e 0} F_{f}(s) F_{F M}(s) \exp (-s \tau)}{s}
$$

is the open loop gain. In Eq. (5) $K_{d c}$ is the loop DC gain, $\phi_{e 0}$ is the steady state phase error given in Eq.(1), $F_{F M}(s)$, $F_{f}(s)$ and $\exp (-s \tau)$ are, respectively, the normalized transfer functions of the slave laser's current FM response, the loop filter and the loop delay. Given the linewidths of the lasers and the loop parameters, the residual phase noise of the locked slave laser can be calculated using Eq. (3).

We have recently conducted the locking experiment on various SCLs[11, 16]. Fig. 2 is a typical power spectrum of the beat signal between the master laser and the locked slave laser. Their frequency is offset by a $850 \mathrm{MHz}$ RF offset signal. From the spectrum we can calculate the RMS differential phase error

$$
\sigma_{\phi_{e}}=\sqrt{\overline{\left(\phi_{e}-\phi_{e 0}\right)^{2}}}=\sqrt{P_{n} / P_{s}}
$$

where $\phi_{e 0}$ is the steady-state phase error given in Eq. (1), $P_{n}$ is the noise power obtained by integrating the measured spectral density over the whole frequency range except the carrier frequency, and $P_{s}$ is the power of the $850 \mathrm{MHz}$ carrier signal. The calculated $\sigma_{\phi_{e}}$ is $\sim 0.12 \mathrm{rad}$.

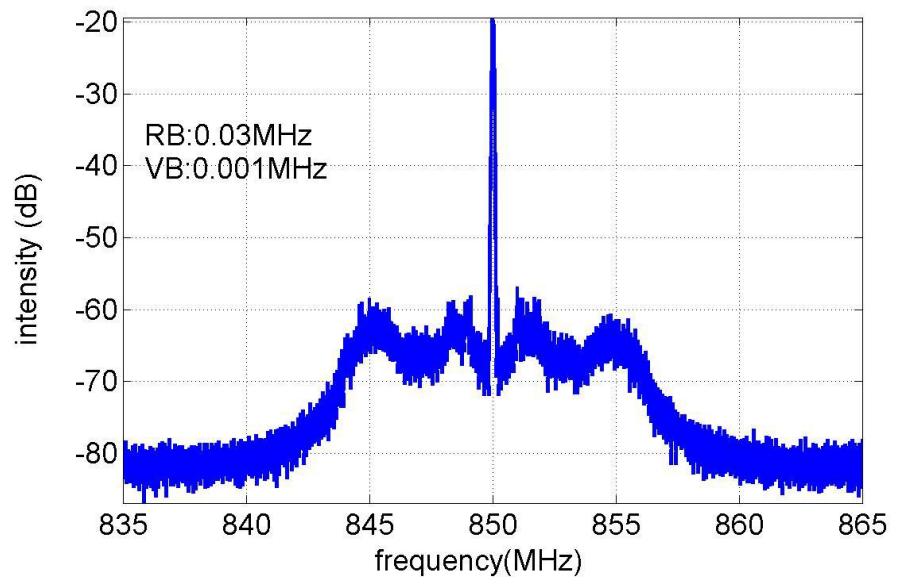

Fig. 2. Typical power spectrum of the locked beat signal between the master laser and the slave laser.

The OPLLs have limited loop bandwidth(BW) due to the non-uniform FM response of SCLs and the loop delay[16-18]. This limited phase tracking BW results in a number of critical issues. First, it leads to the non-negligible residual phase noise between the master laser and the slave laser. Second, it limits the acquisition range (the maximal frequency difference between the free-running slave laser and the master laser for the OPLL to acquire lock) and the holding range (the maximal allowable frequency difference between the free-running slave laser and the master laser for the OPLL to stay in lock). In a first order PLL without any compensation filter, the holding range and acquisition range are simply $K_{d c} / 2 \pi$ [15], which is a few MHz for our systems. The limited acquisition range makes it very difficult for the loop to acquire lock after being turned on. When the holding range is small, the frequency drifting of the SCLs due to thermal, acoustic and electric disturbance can constantly throw the loop out of lock. These issues become more challenging when an array of lasers is to be locked. Compensation circuits and filters are necessary to address these issues.

Fig. 3 shows the schematic diagram of the compensation circuits and filters used to improve the holding and acquisition ranges. A lag-lead filter with transfer function $F(s)=\left(1+\tau_{2} s\right) /\left(1+\tau_{1} s\right)$ can increase the holding range by a factor of $\tau_{1} / \tau_{2}[15,19]$. We first built a passive lag-lead filter (Fig. 3(a)) and it increased the holding range from $\pm 10 \mathrm{MHz}$ to $\pm 200 \mathrm{MHz}$ and the locking time from tens of seconds to one hour. However, any further increase of the factor $\tau_{1} / \tau_{2}$ is limited by the current driving capability of the RF mixer in the phase detector (Fig. 1(a)). We further designed an active lag-lead filter (Fig. 3(b)) which increased the holding range to more than $\pm 3 \mathrm{GHz}$. To increase the acquisition range, an aided acquisition circuit (AAC, Fig. 3(c)) can be used. The AAC compares the frequency of the beat signal with the frequency of the RF offset signal, and generates a current ramp which brings the frequency of the beat signal to be 
within the acquisition range of the OPLL. [11] The use of an AAC circuit in our OPLL improved the acquisition range from $\pm 10 \mathrm{MHz}$ to $\pm 1.1 \mathrm{GHz}$.

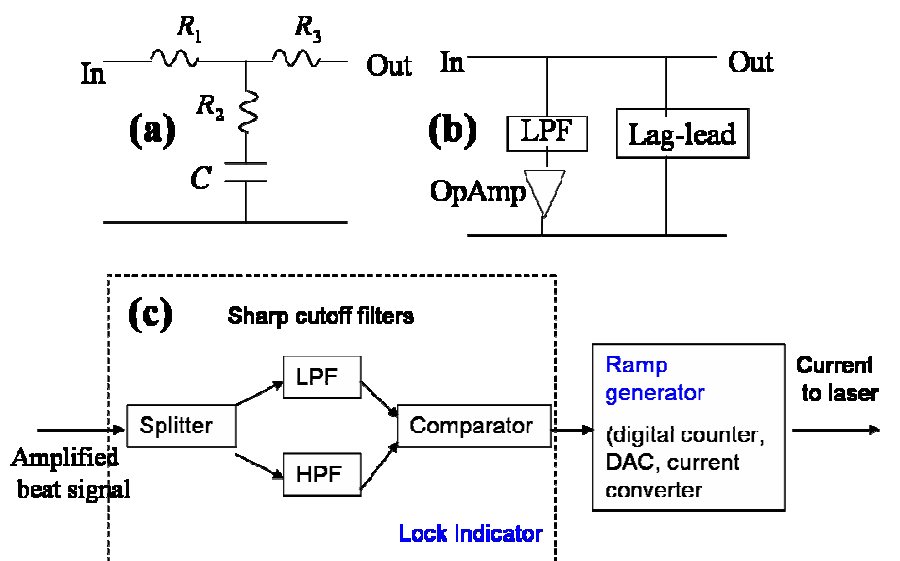

Fig. 3. Schematics of the compensation circuits and filters. (a) passive lag-lead filter; (b) active lag-lead filter; (c) aided acquisition circuit.

\section{COHERENT BEAM COMBINING WITH OPLLS}

\subsection{Filled-aperture $\mathrm{CBC}$ in fiber}
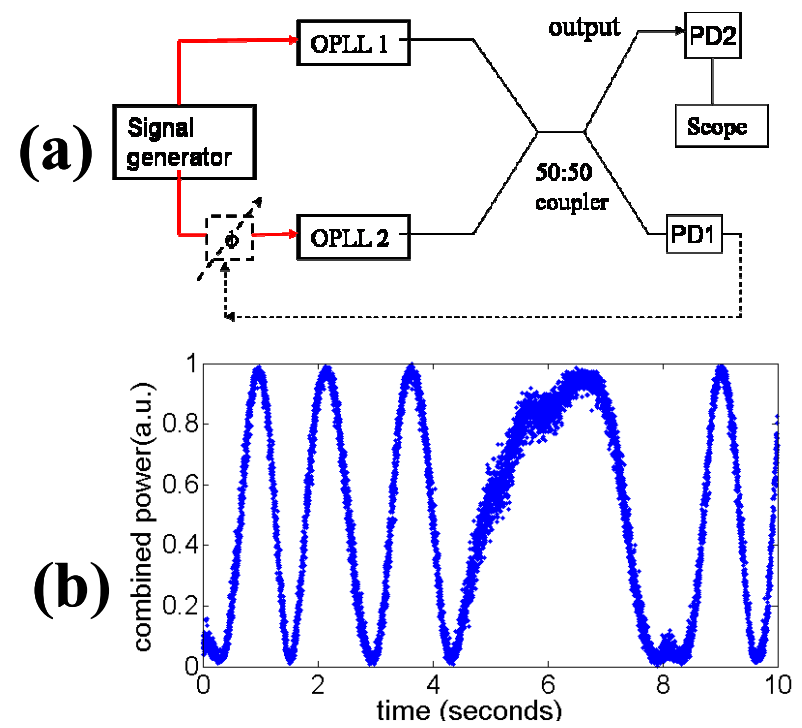

Fig. 4(a). Schematic diagram of combining two OPLLs without or with a RF phase shifter loop. The dotted line stands for the RF phase shifter loop. (b). Measured combined signal(PD2) without the RF phase shifter loop.

As a proof-of-concept demonstration, we first phase-locked two slave lasers to the same master laser and coherently combined their outputs using a fiber directional coupler[11, 16], as illustrated in Fig. 4(a).

The details of OPLL1 and OPLL2 are given in Fig. 1(a) and thus not plotted here. We first conducted the experiment without a RF phase shifter feedback loop. The combined signal is measured and the data captured with an oscilloscope is shown in Fig. 4(b). The slowly varying DC signal shows that the two beams are combined coherently, however their relative phase is drifting on the time scale of seconds due to the differential optical path length variations in the fiber. This slow phase variation needs to be corrected in real time to maintain a constant maximal combined output. This is typically done with an optical phase modulator, a piezo fiber stretcher or an AOM[6-8]. As indicated in Eq. (1), the phase of the slave laser depends on that of the RF offset signal. Thus we can use a RF phase shifter to correct for the optical path length variation as shown by the dotted line in Fig. 4(a). However a phase shifter has limited dynamic range and a complicated phase unwrapping circuit is needed to effective increase its dynamic range. Here we propose using a Voltage controlled oscillator as the RF phase shifter since it acts as a phase integrator and has infinite dynamic range. 


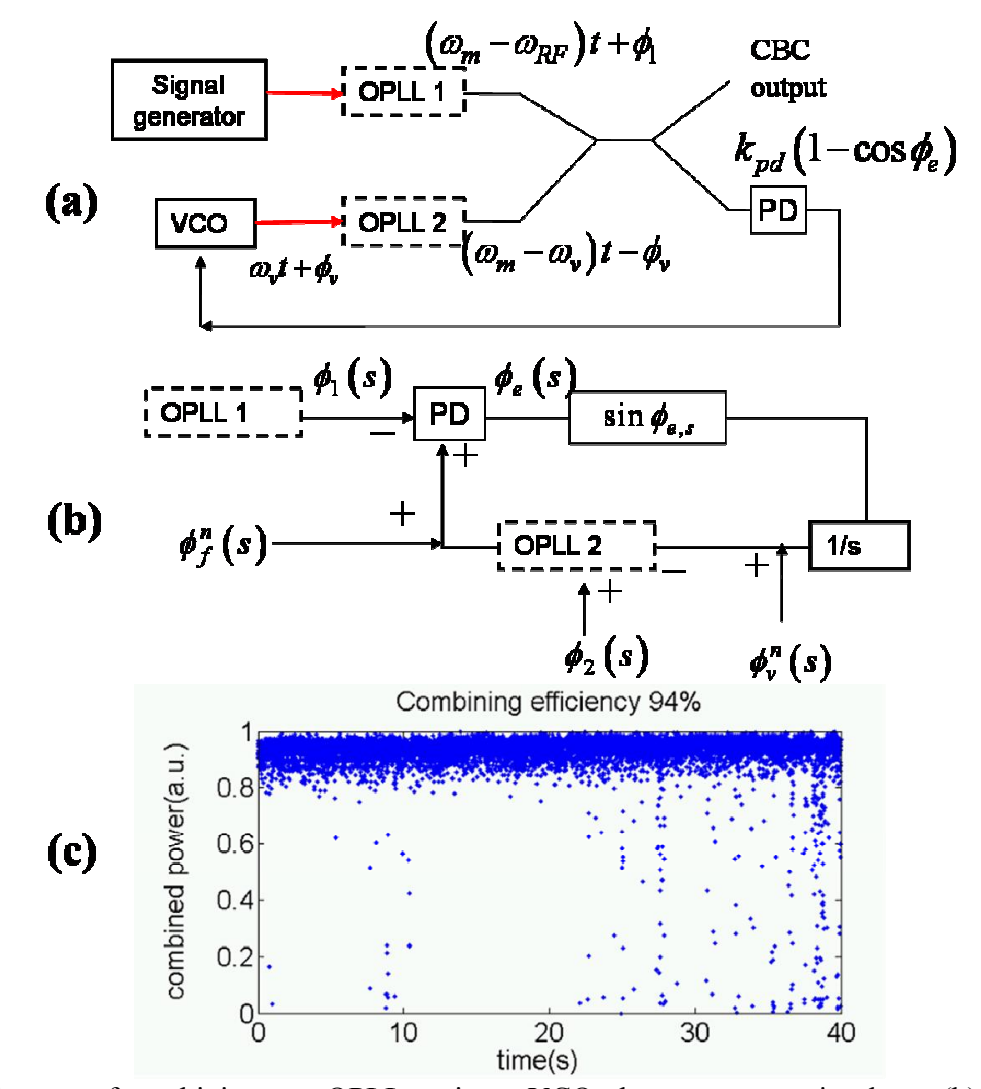

Fig. 5(a) Schematic diagram of combining two OPLLs using a VCO phase compensation loop. (b) Small signal linearized model of the VCO phase compensation loop. (c) Measured combined signal with the optical path length variation being corrected for by the VCO phase compensation loop.

Fig. 5(a) gives a schematic diagram of combining two OPLLs using a VCO phase compensation loop. A rigorous analysis treating OPLL2 and the VCO loop as a coupled system is given in reference[20]. Here we adopt a simplified picture by decoupling the two control loops and studying them separately. This picture is valid when we limit the VCO loop BW to be much smaller than that of OPLL2. In this case we can assume OPLL2 always tracks the phase of the VCO instantly when the VCO loop is correcting for the optical path length variation. The analysis of OPLL2 is already given in Section I. In Fig. 5(a), We observe the VCO loop is similar to a standard PLL except for that here the phase detector output is proportional to $\left(1-\cos \phi_{e}\right)$ instead of $\sin \phi_{e}$. Following the standard PLL analysis[15], the evolution equation of the VCO loop is given by

$$
\left(\omega_{m}-\omega_{v, f}\right) t-K_{v} \int\left(1-\cos \phi_{e}\right) d t-\left(\omega_{m}-\omega_{R F}\right) t-\phi_{1}=\phi_{e}
$$

where $\omega_{m}, \omega_{R F}, \omega_{v, f}$ are respectively the frequency of the master laser, the RF signal generator, and the free-running VCO, $K_{v}$ is the VCO loop gain, $\phi_{e}$ is the phase difference between the two slave laser beams and $\phi_{1}$ is the residual phase noise of beam 1. In obtaining Eq. (7) we have used the equality $\phi_{v}=K_{v} \int\left(1-\cos \phi_{e}\right) d t$ for the VCO output phase. Differentiating Eq. (7) and setting the time derivatives of $\phi_{1}$ and $\phi_{e}$ equal to zero, we find the steady state phase error:

$$
\phi_{e, s}=\cos ^{-1}\left(1-\frac{\omega_{R F}-\omega_{v, f}}{K_{v}}\right)
$$

As long as $0<\left(\omega_{R F}-\omega_{v, f}\right) / K_{v}<2$, Eq. (8) has a solution and the VCO frequency can be locked to the signal generator's frequency. It is important to note that the steady state phase error under lock, $\phi_{e, s}$, which controls the CBC efficiency - can be adjusted by tuning the frequency difference $\omega_{R F}-\omega_{\nu, f}$. High combining efficiency is achieved by 
minimizing $\phi_{e, s}$. However, this comes at the cost of increased cycle-slips caused by the residual phase noise and the frequency jitter of the VCO. The smallest feasible $\phi_{e, s}$ is limited mainly by the frequency jitter of the VCO compared to the loop gain $K_{v}$. The influence of this non-zero $\phi_{e, s}$ on the system performance will be studied in more details in section 4.

The system can again be linearized with a small signal perturbation about the steady-state point. We should note that this linearization is, strictly speaking, inaccurate because Eq. (7) is highly nonlinear close to the null point $\phi_{e, s}=0$. However, a linear analysis is useful to obtain physical insight into the loop performance. A small signal linearized model is presented in Fig. 5(b). $\phi_{f}^{n}(s)$ and $\phi_{v}^{n}(s)$ stand for the optical path length variation in the fiber and the phase noise of the free-running VCO respectively. $\phi_{1}$ and $\phi_{2}$ contain the residual phase noise of OPLL1 and OPLL2 pointed out in section 2 (Eq. (3)). Following the PLL analysis we obtain

$$
\phi_{2}(s)-\left[\frac{K_{v} \sin \phi_{e, s}}{s} \cdot \phi_{e}(s)+\phi_{v}^{n}(s)\right]-\phi_{1}(s)+\phi_{f}^{n}(s)=\phi_{e}(s)
$$

Solving for $\phi_{e}(s)$ gives

$$
\phi_{e}(s)=\frac{\phi_{2}(s)+\phi_{f}^{n}(s)-\phi_{v}^{n}(s)-\phi_{1}(s)}{1+K_{v} \sin \phi_{e, s} / s}
$$

In Eq. (10) we first observe that a nonzero $\phi_{e, s}$ is needed to provide the small signal loop gain. Second, the residual phase noises from OPLL1 and OPLL2 are mostly concentrated at frequencies of a few MHz as indicated by the spectral shoulder in Fig. 2. While the BW of the VCO loop is much smaller than MHz and hence doesn't affect the residual phase noise of OPLL1 and OPLL2. A typical high quality VCO has very low phase noise. The optical path length variation $\phi_{f}^{n}(s)$ is also at very low frequency $(\sim \mathrm{Hz})$. The noise from these sources can be significantly suppressed by the VCO loop with a BW of $\sim 100 \mathrm{kHz}$. Therefore the residual phase noise of OPLL1 and OPLL2 will remain the main noise source of $\phi_{e}$.

We performed the CBC experiment depicted in Fig. 5(a) using two 1064nm commercial SCLs. Fig. 5(c) shows the measured combined output. Compared to Fig. 4(b), we see that the phase variation in the fiber is corrected and the combined power stays constant. From the data we calculate the CBC efficiency to be about $94 \%$. The reduction of the CBC efficiency can be attributed mainly to the non-zero steady-state locking point of the VCO loop and the residual phase noise of the OPLLs. A systematic analysis of the CBC efficiency will be given in section 4 .

\subsection{Tiled-aperture CBC with OPLLs}

The OPLLs technology can also be applied to the tiled-aperture CBC approach. Compared to the filled-aperture approach, the tiled-aperture approach is more tolerant to the failure of an individual laser and enables the possibility of electronic beam steering. Fig. 6(a) plots the schematic diagram of the tiled-aperture CBC experiment utilizing OPLLs. The signals of the master laser and the slave lasers are expanded with fiber optical collimators and combined using a beam splitter of $0.25 \%$ reflectivity. Two free space photodetectors are used to detect the phase difference between the master laser and the slave lasers. The photocurrent is mixed with a RF offset signal, and the down-converted phase error signal is fed back to the slave laser to complete the loop. The two slave laser beams become coherent after they are phase locked to a common reference phase plane provided by the master laser beam with a common frequency and phase offset provided by the RF signal. In this heterodyne OPLL scheme, a RF phase shifter can be used to adjust the relative phase between the individual slave lasers. $99.75 \%$ of the slave lasers' signals are transmitted through the beam splitter and combined using a focus lens. An IR camera is placed at the focus plane to measure the intensity profile of the combined beam.

In Fig. 6(b)-(f) we plot the measured intensity profiles of the individual beams, the incoherently combined beam and the coherently combined beam acquired by the IR camera. When the two slave lasers are both locked to the master laser with the same offset frequency, an interference fringe appears in the image(Fig. 2(d)-(e)). In this case even when we disturb the fiber connecting the slave laser with the fiber collimator, the fringe is stable because the OPLL always locks the slave laser beam to the master laser beam at the plane of the photodetector. We also use a RF phase shifter to change the phase of an individual laser beam. In Fig. (2d), the two beams are out of phase and a dark stripe is produced in the 
center. After adjusting the phase shifter by $180^{\circ}$, a bright stripe is generated in the center and we essentially realize the beam steering electronically.

In Fig. 6(c) we further plot the intensity profiles of the IR images along a horizontal section through the brightest point in the images. When the two beams are coherently combined, the intensity profile narrows and the peak value almost doubles. The Strehl ratio is about 0.9 , which is limited by the polarization mismatch and the residual phase fluctuation between the two slave lasers.
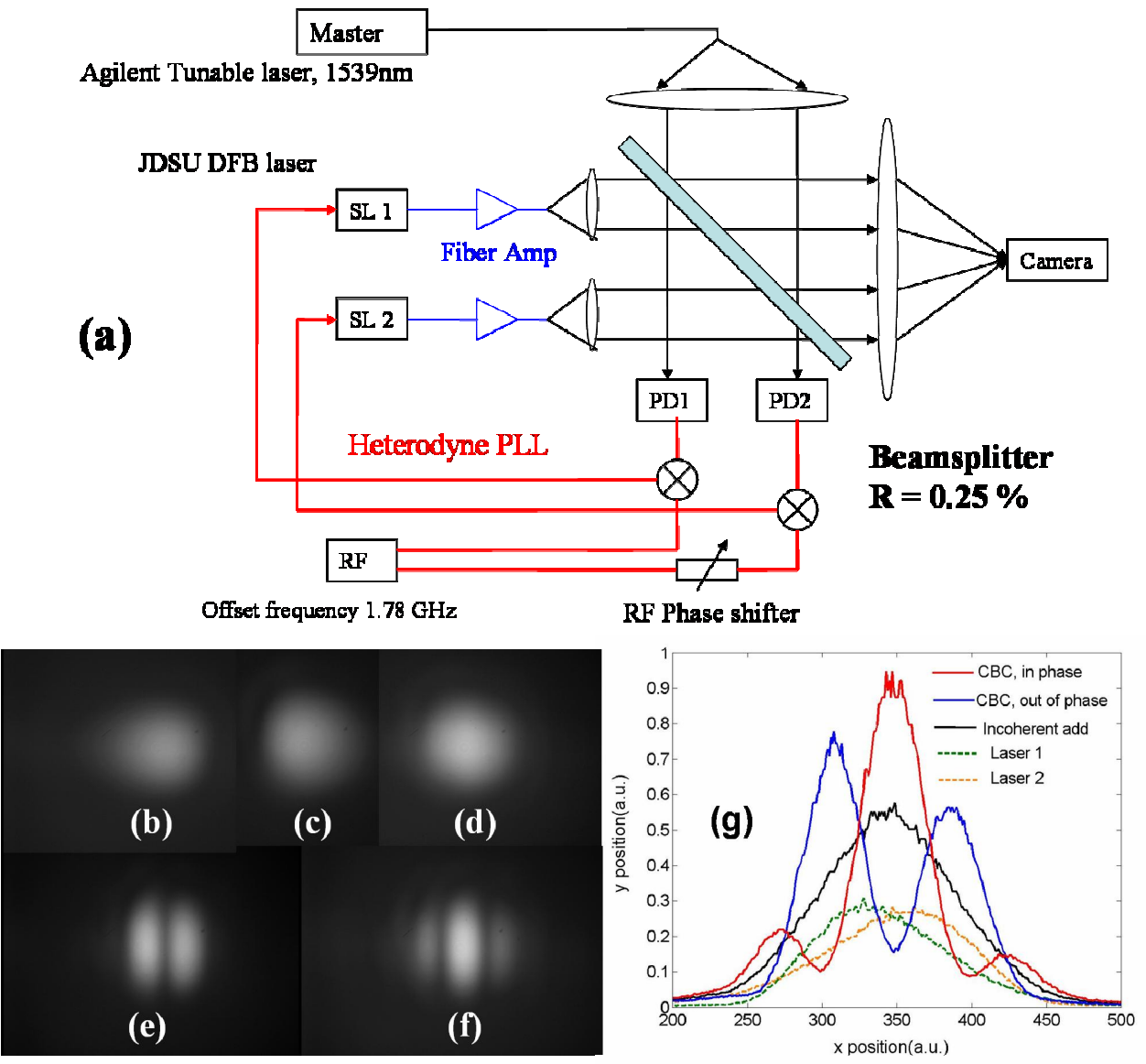

Fig. 6(a) Schematic diagram of a tiled-aperture coherent beam combining system using OPLLs.

(b) - (f): IR camera images of (b) laser beam1, (c) laser beam2, (d) incoherently combined beam, (e) out of phase coherently combined beam and (f) in phase coherently combined beam. (g) Intensity profiles along horizontal sections of the IR images (b) $-(\mathrm{f})$.

\section{ANALYSIS OF THE COMBINING EFFICIENCY}

The efficiency of combining a large number of beams will be degraded by various noise sources. The OPLLs used for the phase control will introduce noises such as the residual phase noise, intensity noise etc. In this section we want to analyze the scalability of the $\mathrm{CBC}$ system under the influence of the various sources of noise. In particular, we will consider the residual phase noise of the OPLLs, the non-zero steady-state phase error in the VCO loops, the intensity noise of the beams, and the phase noise added by the fiber amplifier. The effect of the phase noise on side by side tiledaperture implementation has been analyzed in reference[21]. Here we use a binary-tree filled-aperture scheme as the example in the analysis.

In a filled-aperture CBC system, $N$ mutually coherent beams can be combined at a beam splitter, or fiber-optic coupler. The simplest example is two plane waves with the same frequency are incident upon a partially reflecting mirror having an amplitude reflectivity $r=1 / \sqrt{2}$. If the two beams have exactly the same amplitude and phase, on the output sides of 
the beam splitter there are two pairs of waves propagating at right angles with intensity of $I_{P 1}=2 I_{0}, I_{P 2}=0$ and the CBC efficiency is $100 \%$. When the phase noise and intensity noise are present, the combining efficiency drops to

$$
\eta=\frac{\overline{I_{p}}}{\overline{I_{1}}+\overline{I_{2}}} \approx 1-\frac{1}{4} \overline{\phi^{2}}-\frac{1}{8}\left(\overline{r_{1}^{2}}+\overline{r_{2}^{2}}\right)
$$

where $r_{1}, r_{2}$ stand for the Relative Intensity Noise (RIN) of beams 1 and 2, and $\phi$ represents the relative phase error between the two beams. In Eq. (11) we also assume the noises have zero mean and are small enough that higher order expansion terms can be ignored.

\subsection{Effect of the OPLL residual phase noise}

We first analyze the influence of the residual phase noise of OPLLs. We use $\phi_{i}(t)$ to represent the phase noise of beam $i$ referred to a common reference plane. The complex amplitude of the corresponding field is $E_{0} e^{i \phi_{i}}$. Adding up all the beams the combined field is

$$
E_{t}=E_{0} \sum_{i=1}^{N} e^{i \phi_{i}}
$$

The intensity is the square of the absolute value of the field. Normalizing the combined power by the total input power, we obtain the $\mathrm{CBC}$ efficiency

$$
\eta=\frac{1}{N^{2}} \overline{\sum_{i, j=1}^{N} e^{i\left(\phi_{i}-\phi_{j}\right)}}=1-\frac{N-1}{N}\left(1-e^{-\sigma^{2}}\right)
$$

In calculating Eq. (13) we have assumed that $\phi_{i}$ obeys Gaussian distribution with zero mean and variance $\overline{\phi_{i}^{2}(t)}=\sigma^{2}$, $\phi_{i}$ and $\phi_{j}$ are uncorrelated, so that $\overline{e^{i\left(\phi_{i}-\phi_{j}\right)}}=\delta_{i, j} e^{-\sigma^{2}}[21,22]$. For $\sigma^{2}<<1$, Eq. (13) reduces to

$$
\eta=1-\frac{(N-1)}{N} \sigma^{2}
$$

As we can see, the combining efficiency converges to $1-\sigma^{2}$ for a large number of beams. For a given number of beams $N$ and a desired combination efficiency $\eta$, the RMS phase error has to satisfy

$$
\sigma \leq \sqrt{(1-\eta) \frac{N}{N-1}}
$$

E.g, if we use $\mathrm{N}=8$ and $\eta=95 \%$, the RMS phase error has to be smaller than 0.24rad . In Fig. 2 the RMS phase error is as small as $0.12 \mathrm{rad}$.

\subsection{Effect of the non-zero steady-state phase error of the VCO loops}

In section 2 we discussed the servo system using a VCO to correct for the optical path-length variations in fiber. We pointed out that a nonzero steady state phase error between the element beams is required to tolerate the frequency jitter of the VCO and to provide a non-zero small signal loop gain, thereby reducing the combining efficiency. In this section we evaluate the effect of this nonzero steady-state phase error on the combining efficiency of a filled-aperture combining system.

We assume that the frequency jitter of the free-running VCO obeys Gaussian distribution with zero mean and rms value $\sigma_{\omega}$. In Eq. (8), if the quantity $\omega_{R F}-\omega_{v, f}$ becomes negative the VCO loop will lose lock. If we set the steady state phase error between the element beams equal to $\left(\omega_{R F}-\omega_{v, f}\right)_{s s}=x \sigma_{\omega}$, the probability that the quantity $\omega_{R F}-\omega_{v, f}$ takes a negative value is described by the cumulative distribution function of Gaussian distribution

$$
F(-x ; 0,1)=\frac{1}{\sqrt{2 \pi}} \int_{-\infty}^{-x} \exp \left(-\frac{u^{2}}{2}\right) d u
$$

In the binary-tree cascaded filled-aperture scheme shown in Fig. 7(a), a VCO loop is needed at each stage where two beams are combined. If any one of the VCO loops loses lock, the whole system is disrupted. If $N=2^{n}$ beams are to be combined, the number of VCO loops will be $2^{n}-1$ and the probability that the whole system stays in lock is hence

$$
P_{\text {lock }}=[1-F(-x ; 0,1)]^{2^{n}-1}
$$


Moreover, the combining efficiency is reduced because of the non-zero value of $\phi_{e, s}$ even if the system is in lock. At each combining level of the binary-tree scheme, the combining efficiency is given by $\left(1+\cos \phi_{e, s}\right) / 2$ with $\cos \phi_{e, s}=1-x \sigma_{\omega} / K_{v}$. The system has $n$ levels therefore the overall combining efficiency when the system stays in lock is

$$
\eta_{\text {lock }}=\left(1-\frac{x \sigma_{\omega}}{2 K_{v}}\right)^{n}
$$

Now we take into account the fact that the system loses lock for sometime, the overall combining efficiency should be the product of $P_{\text {lock }}$ and $\eta_{\text {lock }}$

$$
\eta=P_{\text {lock }} \cdot \eta_{\text {lock }}=[1-F(-x ; 0,1)]^{2^{n}-1} \cdot\left(1-\frac{x \sigma_{\omega}}{2 K_{v}}\right)^{n}
$$

$P_{\text {lock }}$ is a monotonously increasing function of $x$ while $\eta_{\text {lock }}$ is a monotonously decreasing function of $x$ in the range $0<x \sigma_{\omega} / K_{v}<2$ where Eq. (8) has a solution. Hence an optimal value of $x$ can be chosen to maximize the efficiency described by Eq. (19).
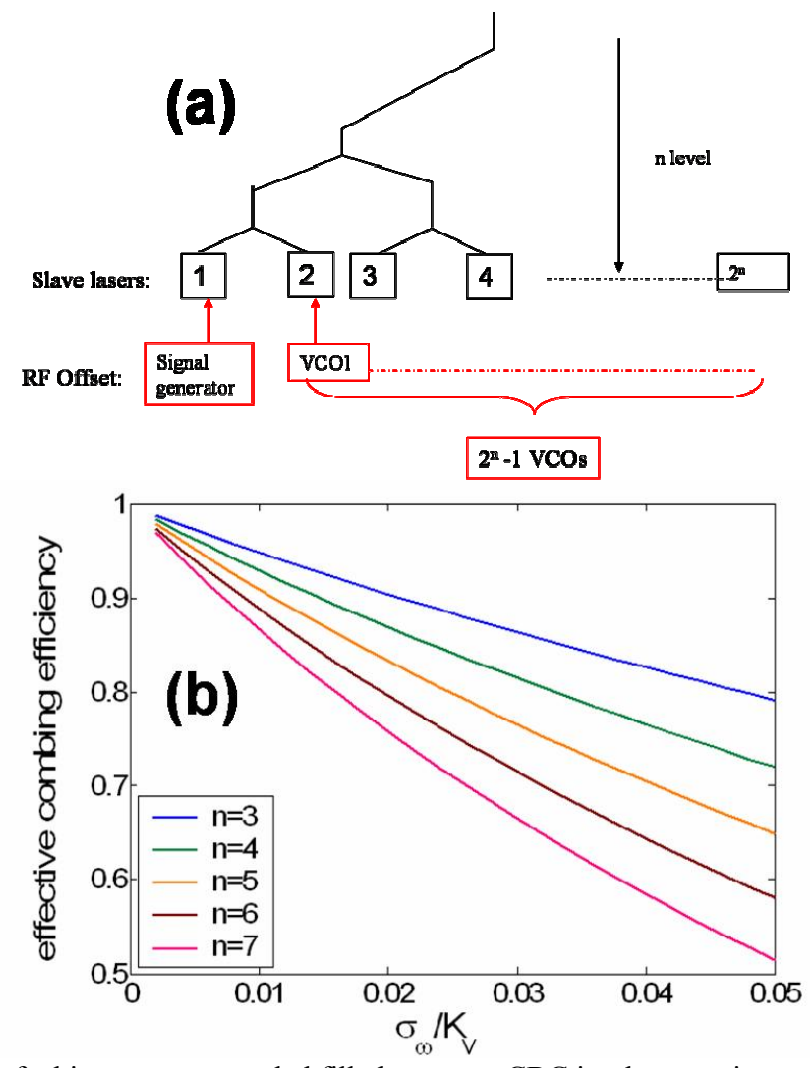

Fig. 7(a) Schematic diagram of a binary-tree cascaded filled-aperture CBC implementation using OPLLs(b)Maximal combining efficiency limited by the normalized VCO frequency jitter $\sigma_{\omega} / K_{v}$. The number of element beams is $2^{n}$.

In Fig. 7(b) we plot the maximal combining efficiency as a function of the normalized frequency jitter $\sigma_{\omega} / K_{v}$ for different values of $n$. The combining efficiency drops quickly with the increase of both $\sigma_{\omega} / K_{v}$ and $n$. Therefore, it is critical to reduce $\sigma_{\omega} / K_{v}$ in order to achieve a high combining efficiency while combining a large number of beams. In our proof-of-concept experiment with one VCO loop, the combining efficiency is around $94 \%$ and we estimate $\sigma_{\omega} / K_{v}$ to be about 0.03 . To improve the combining efficiency, one solution is to reduce $\sigma_{\omega}$, i.e. use a "pure" tone VCO with a smaller frequency jitter. Another solution is to increase $K_{v}$, which determines the VCO loop gain and is ultimately 
limited by the loop delay particularly if a fiber amplifier is to be used. This can be done by using a lag-lead filter similar to the filter design discussed in section 2.

\subsection{Effect of intensity noise}

The combining efficiency can also be degraded by intensity noise, as indicated in Eq. (11). The free-running RIN of the laser is typically very small compared to the phase noise. However, when the slave laser is in the OPLL, the feedback current used to control the frequency and phase of the slave laser can add intensity noise. This added intensity noise can be estimated as follows. Assume the current fed back into the SCL is $i=i_{0} \sin \phi_{e}$, where $\phi_{e}$ is the differential phase error, then the RIN is

$$
r_{s}=\Delta P / P_{0}=\frac{K_{a m}}{P_{0}} i_{0} \sin \phi_{e}
$$

where $P_{0}$ is the steady state power and $K_{a m}$ is the amplitude modulation coefficient of the laser. $K_{a m}$ can be estimated from the slope of the laser's P-I curve

$$
K_{a m}=P_{0} /\left(I-I_{t h}\right)
$$

Use the SCLs in our OPLLs as an example, $I-I_{t h} \approx 300 \mathrm{~mA}$ and $i_{0}$ is measured to be smaller than $1 \mathrm{~mA}$. Substituting the numbers Eq. (20) becomes $r_{s}(t)=\sin \phi_{e}(t) / 300$, and the added RIN in this case is more than two orders of magnitude smaller than the residual phase noise. Thus it is safe to neglect the effect of the intensity noise.

\subsection{Phase noise added by the fiber amplifier}
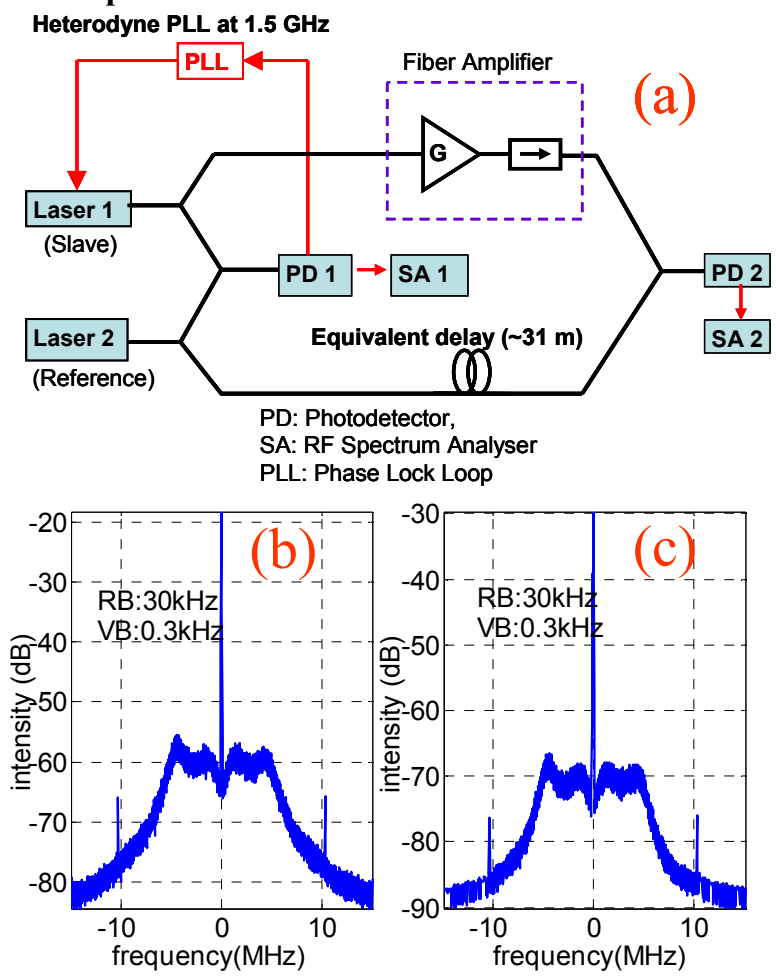

Fig. 8 (a). Experimental setup to measure the fiber amplifier phase noise added to the OPLL. (b) and (c). Beat spectra at the photodetectors PD1 and PD2 in (a).

To achieve an average power of above a $\mathrm{kW}$, tens of thousands of SCLs need to be combined, which is very difficult to realize. A more practical option is to use the locked slave SCLs to seed tens of high power fiber amplifiers, whose output beams are then coherently combined. Since CBC is very sensitive to phase noise, the phase noise introduced by the fiber amplifiers needs to be examined. 
Here we measure and compare the beat signal between the master laser and the slave laser with and without a fiber amplifier as shown in Fig. 8(a). The fiber amplifier is a $1064 \mathrm{~nm}$ Nufern $3 \mathrm{~W}$ Yb-doped fiber amplifier. A balanced interferometer is used to remove the phase noise of the master laser[23]. Comparing the beat signal before and after the fiber amplifier, we don't see any additional phase noise added by the amplifier. In fact, the additive phase noise due to the ASE in the fiber amplifier is far below our measurement sensitivity and can be safely neglected compared to the other factors reducing the combining efficiency. While the amplifier does add a lot of thermal phase variation[7], and picks up some acoustic noise, they are at very low frequency compared to the bandwidth of the VCO phase compensation loops and should be significantly suppressed by the VCO loops.

\section{CONCLUSION}

We have presented a detailed study of a control system using OPLLs to coherently combine optical beams. In the preliminary demonstration, we have successfully combined two commercial SCLs using both the filled-aperture approach and the tiled-aperture approach. A promising combining efficiency of $94 \%$ is achieved with the filled-aperture scheme. If the tiled-aperture scheme is to be used, the less-than-unity fill factor remains a critical issue[2]. If the filledaperture scheme is to be used, the loss caused by the phase error in the VCO loops scales up as $\sim \log _{2} N$. This poses a serious challenge if $N$ is very large. However, with single-mode fiber amplifier power reaching kilowatts, combining only tens of beams can scale the power up to more than $10 \mathrm{~kW}$ or even $100 \mathrm{~kW}$. Using the filled-aperture scheme and the OPLLs described in this paper, our collaborators have achieved a single coherent beam of hundreds of Watts by combining four element beams.

\section{ACKNOWLEDGEMENT}

The authors wish to acknowledge the support of DARPA's MTO office (J. Mangano).

\section{REFERENCES}

1. L. Bartelt-Berger, U. Brauch, A. Giesen, H. Huegel, and H. Opower, "Power-scalable system of phase-locked single-mode diode lasers," Applied Optics 38(27), 5752-5760 (1999).

2. T. Y. Fan, "Laser beam combining for high-power, high-radiance sources," Ieee Journal of Selected Topics in Quantum Electronics 11(3), 567-577 (2005).

3. T. M. Shay, V. Benham, J. T. Baker, B. Ward, A. D. Sanchez, M. A. Culpepper, D. Pilkington, J. Spring, D. J. Nelson, and C. A. Lu, "First experimental demonstration of self-synchronous phase locking of an optical array," Optics Express 14(25), 12015-12021 (2006).

4. A. Shirakawa, T. Saitou, T. Sekiguchi, and K. Ueda, "Coherent addition of fiber lasers by use of a fiber coupler," Optics Express 10(21), 1167-1172 (2002).

5. Y. Kono, M. Takeoka, K. Uto, A. Uchida, and F. Kannari, "A coherent all-solid-state laser array using the Talbot effect in a three-mirror cavity," Ieee Journal of Quantum Electronics 36(5), 607-614 (2000).

6. B. A. Demoustier S, lallier E and Huignard J.P., "Coherent Combining of 1.5um Er-Yb Doped Single Mode Fiber Amplifier," (2006).

7. S. J. Augst, T. Y. Fan, and A. Sanchez, "Coherent beam combining and phase noise measurements of ytterbium fiber amplifiers," Optics Letters 29(5), 474-476 (2004).

8. C. X. Yu, J. E. Kansky, S. E. J. Shaw, D. V. Murphy, and C. Higgs, "Coherent beam combining of large number of PM fibres in 2-D fibre array," Electronics Letters 42(18), 1024-1025 (2006).

9. L. H. Enloe and J. L. Rodda, "Laser Phase-Locked Loop," Proceedings of the Institute of Electrical and Electronics Engineers 53(2), 165-166 (1965).

10. L. N. Langley, M. D. Elkin, C. Edge, M. J. Wale, U. Gliese, X. Huang, and A. J. Seeds, "Packaged semiconductor laser optical phase-locked loop (OPLL) for photonic generation, processing and transmission of microwave signals," Ieee Transactions on Microwave Theory and Techniques 47(7), 1257-1264 (1999).

11. W. Liang, N. Satyan, A. Yariv, A. Kewitsch, G. Rakuljic, F. Aflatouni, H. Hashemi, and J. Ungar, "Coherent power combination of two Master-oscillator-power-amplifier (MOPA) semiconductor lasers using optical phase lock loops," Optics Express 15(6), 3201-3205 (2007).

12. S. Kobayashi, Y. Yamamoto, M. Ito, and T. Kimura, "Direct Frequency-Modulation in AlGaAs SemiconductorLasers," Ieee Journal of Quantum Electronics 18(4), 582-595 (1982).

13. A. Yariv, "Dynamic analysis of the semiconductor laser as a current-controlled oscillator in the optical phasedlock loop: applications," Optics Letters 30(17), 2191-2193 (2005). 
14. R. C. Steele, "Optical Phase-Locked Loop Using Semiconductor-Laser Diodes," Electronics Letters 19(2), 6971 (1983).

15. F. M. Gardner, Phaselock Techniques, third ed. (Wiley, 2005).

16. W. Liang, A. Yariv, A. Kewitsch, and G. Rakuljic, "Coherent combining of the output of two semiconductor lasers using optical phase-lock loops," Optics Letters 32(4), 370-372 (2007).

17. R. T. Ramos and A. J. Seeds, "Delay, Linewidth and Bandwidth Limitations in Optical Phase-Locked Loop Design," Electronics Letters 26(6), 389-391 (1990).

18. P. Correc, O. Girard, and I. F. Defaria, "On the Thermal Contribution to the Fm Response of Dfb Lasers Theory and Experiment," Ieee Journal of Quantum Electronics 30(11), 2485-2490 (1994).

19. D. R. Stephens, Phase-Locked Loops For Wireless Communications (Kluwer Academic Publishers, 1998).

20. W. L. Naresh Satyan, Firooz Aflatouni, Amnon Yariv, Anthony Kewitsch, George Rakuljic, and Hossein Hashemi, "Phase-controlled Apertures using Heterodyne Optical Phase-Lock Loops " (submitted to Photonics Technology Letters).

21. C. D. Nabors, "Effects of Phase Errors on Coherent Emitter Arrays," Applied Optics 33(12), 2284-2289 (1994).

22. D. S. Elliott, R. Roy, and S. J. Smith, "Extra-Cavity Laser Band-Shape and Bandwidth Modification," Physical Review A 26(1), 12-18 (1982).

23. E. Rochat and R. Dandliker, "New investigations on the effect of fiber amplifier phase noise," Ieee Journal of Selected Topics in Quantum Electronics 7(1), 49-54 (2001). 